American J. of Engineering and Applied Sciences 4 (4): 485-490, 2011

ISSN 1941-7020

(C) 2014 G.M. Tosi Beleffi et al., This open access article is distributed under a Creative Commons Attribution

(CC-BY) 3.0 license

\title{
Metro Access Convergence for Broadband Future Low Energy Consumption Networks
}

\author{
${ }^{1}$ Tosi Beleffi, G.M., ${ }^{2}$ E. Leitgeb, ${ }^{3}$ G. Incerti, ${ }^{3}$ S. Di Bartolo, \\ ${ }^{3}$ V. Carrozzo, ${ }^{4}$ A.L.J. Teixeira, ${ }^{5}$ S. Aleksic and ${ }^{5}$ A. Lovric \\ ${ }^{1}$ Deparment of MISE-Communication, \\ Superior Institute of Communications, Rome, Italy \\ ${ }^{2}$ University of Graz, Graz, Austria \\ ${ }^{3}$ University of Tor Vergata, Faculty of Electronic Engineering, \\ Department of Communications, Rome, Italy \\ ${ }^{4}$ Universidade de Aveiro, Instituto de Telecomunicacoes, Aveiro, Portugal \\ ${ }^{5}$ Institute of Communications Networks, \\ Vienna University of Technology, Vienna, Austria
}

\begin{abstract}
Problem statement: In study the impact of the optical technologies and infrastructures on the reduction of the carbon footprint maintaining high level of broadband to the end user is discussed. Approach: Authors analyze the main energy consumers in Core, Metro and Access Networks as well as the topology and the performaces of the EU FP7 SARDANA Project, a long reach full optical metro access convergent network. This permit to have a clear and innovative view on the topic of the green networks comparing commercial and on the edge solutions for the critical access segment. Three scenarios have been assumed with different uplink bandwidths: unlimited uplink in the $\mathrm{CO}$, limited uplink of $400 \mathrm{Gbit} \mathrm{sec}^{-1}$ and strong limitation of $100 \mathrm{Gbit} \mathrm{sec}^{-1}$. Additionally, three different sizes of the access network with $100,1,000$ and 10,000 subscribers connected to a single CO have been considered. Results: Increasing the number of users and reducing the uplink bandwidth, the difference in energy efficiency between TDM and PONs and P-t-P FTTH networks increases. Conclusion: Authors conclude that in general, a SARDANA like network provides very good energy efficiency for both limited and unlimited uplink and for small and large networks.
\end{abstract}

Key words: Time Domain Multiplexing (TDM), Visual Networking Index (VNI), Peer-to-Peer (P2P), Information and Communication Technology (ICT), Local Area Networks (LAN), Personal Computers (PC), Information Technology (IT), Wavelength Domain Multiplexing (WDM), Gigabit Ethernet Passive Optical Networks (G/EPON)

\section{INTRODUCTION}

Internet usage has accelerated dramatically in recent years, pressing network operators to seek innovations that will enable them to profitably meet consumers' and businesses' demand for high-speed Internet bandwidth. All evidence indicates that this trend will not only continue, but most likely will grow. Although file-sharing and Peer-to-Peer (P2P) networking are still the largest drivers of consumer bandwidth usage (46 and 39\% respectively, in 2009), the Visual Networking Index (VNI) forecast from CISCO indicates they are being eclipsed by video and other types of content. Internet video traffic will surpass peer-to-peer traffic by the end of the year (2010) marking the first time that $\mathrm{P} 2 \mathrm{P}$ is not the largest Internet traffic type. By 2014, the forecast says, 91\% of Internet traffic will be video and consumers' share of global IP traffic will raise from $79-87 \%$. On the other side, the carbon footprint is among the hot topics of discussion in last year's: a recent report estimates that the urban population will increase from $3.3 \mathrm{~B}$ (2007)-6.4 B (2050). Thus, in the next years we will observe a rural to urban migration or a rural to urban transformation that will lead to a presence of $70 \%$ of population in major cities by 2050 . This prediction can have extra impact if correlated with the fact that $75 \%$ of the world energy is consumed in the cities. Focusing

Corresponding Author: Tosi Beleffi, G.M., Department of MISE-Communication, Superior Institute of Communications, Rome, Italy 
attention on the Information Technology (IT) sector, the total amount of $\mathrm{CO}_{2}$ emissions from this industry could amount to $2 \%$ of global carbon emissions. Changing the perspective from product to service, using the Information and Communication Technology (ICT) power, is possible to drastically reduce the carbon emission. Virtualization, dematerialization and activities delocalization, infect, are only few examples of the possible implementable solutions. Obviously emissions caused from the IT sector should not be ignored, but the emphasis on reducing the emissions from the sector itself should be proportional to the potential for reductions through services provided.

The telecom sector where the opportunities through the services provided hold such a reduction potential as for the IT industry. Telecommunications industry may have a crucial role in answering the challenges stemming from increased globalization.

The study is organized as follows. In Material and Methods authors report on the main energy consumers in Core, Metro and Access networks as well as on the topology and performances of the EU FP7 SARDANA Project. In Results measurements and comparison of energy efficiency between different choices are reported. Last but not least the Discussions and Conclusions where the main results are outlined.

\section{MATERIALS AND METHODS}

A new convergence: It is a fact that the proliferation of broadband networks has been and, in the near future "will be", driven by new multimedia bandwidth hungry services. Nowadays, EU aims to obtain a wide participation of the knowledge society, announcing the will to give $100 \mathrm{Mbps}$ access to $50 \%$ of the EU population. Thus several European projects are facing and studying the way to increase the bandwidth to the end users lowering simultaneously costs and power consumption.

To satisfy the customers, TLC operators are pushed to apply for geo-marketing studies to understand which and where the best technologies/techniques must or should be adopted. Accordingly to recent studies, it must be pointed out that the current power consumption of the worldwide network, depending on several factors, is at $2.4 \%$ of the global electricity consumption; the bandwidth hungry sectors are leaded by: the Access, the Mobile and the Data Centers (Aleksic, 2009). In Core and Metro Networks the most power consuming element is the router (packet processor and routing engine) while, in the Access and Local Area Networks (LAN), the presence of Personal Computers (PC), Servers, Data Centers represent real points of energy consumption.
Adopting optical processing at wavelength and/or packet level, hybrid switching matrix, pushing the fiber close to the end-user Fiber To The-X -> Fiber To The Home (FTTx $->$ FTTH), increasing the efficiency in the transport technology and protocols can be possible solutions to lower the overall energy consumption. In this list of the opportunities, FTTH with Passive Optical Networks (PONs) infrastructures can be a solution that, in the wired world, conciliate the requirements of the end user, offering highest data rates, with the will of governments assuring lowest energy consumption $(2,5)$. The SARDANA network, Fig. 1, transparently merges Time Domain Multiplexing (TDM) single-fiber passive tree sections with a main Wavelength Domain Multiplexing (WDM) double-fiber ring by means of passive Remote Nodes (RN), as shown in Fig. 1. The $100 \mathrm{~km}$ WDM ring transports 32 wavelengths for $>1000$ users, for a TDM tree splitting ratio of 1: 32 and only 1 wavelength per TDM tree. Network protection and traffic balancing properties are provided by the ring configuration and the resilient design of the RNs, guarantying always a connection between each RN and the Central Office (CO) even in the case of fiber cut. The WDM ring is implemented by a double-fiber to avoid main Rayleigh Backscattering (RB) impairments. Bidirectional propagation takes place at the single-fiber TDM trees.

The wavelength transparency of the ring and Optical Network Units (ONUs) and the wavelength add/drop feature of the RNs enables sharing of the same network infrastructure, until the RNs or even up to the ONT/ONU, by several operators and allows users to select the operator by easily exchangeable filters at the ONU.

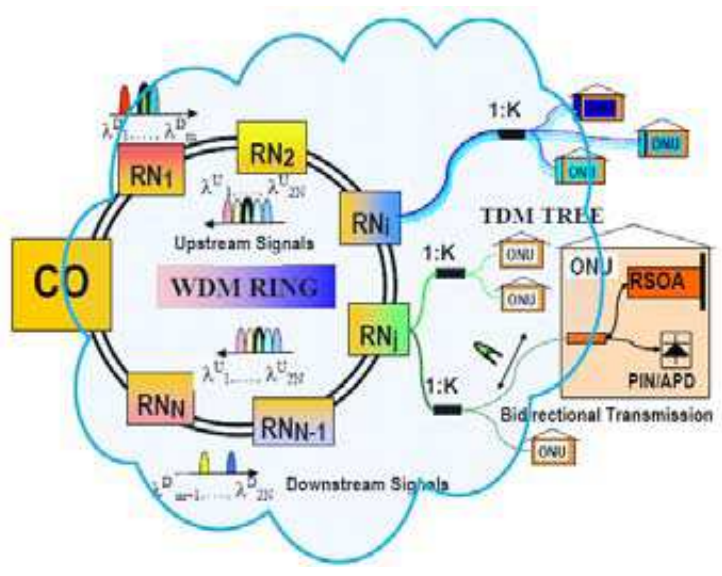

Fig. 1: A long reach broadband passive infrastructure (EU FP7 SARDANA Project) for metro access convergence implementation 
Am. J. Engg. \& Applied Sci., 4 (4): 485-490, 2011

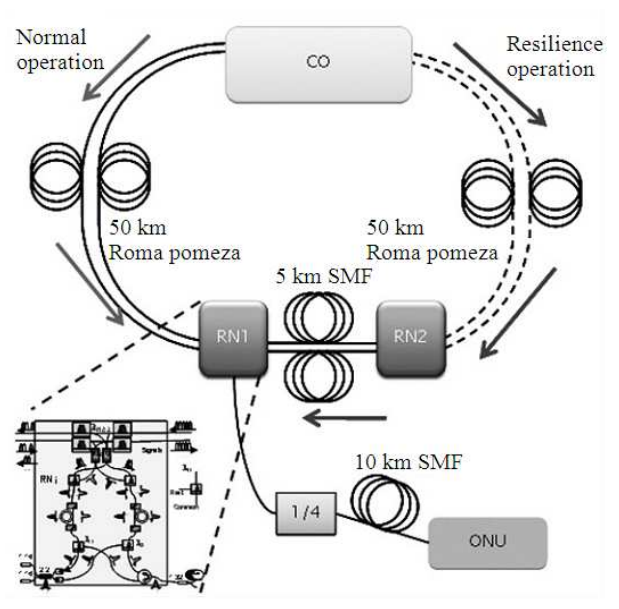

Fig. 2: Network test configuration with $105 \mathrm{~km}$ ring by using an installed optical cable between Rome and Pomezia; RN scheme (inset)
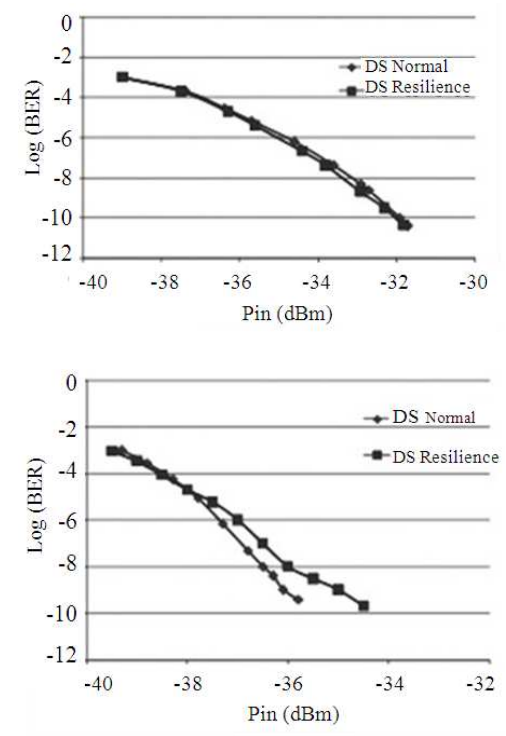

Fig. 3: DS (above)/US (below) transmission BER measurements

This way, SARDANA offers a possible solution for implementing multi-operability in the physical layer, by simply allocating a set of wavelength Downstream/Upstream channels to each operator.

The WDM/TDM overlay in the SARDANA network eases the migration process from legacy PON solutions, such as standard Gigabit Ethernet Passive Optical Networks (G/EPON) and next-generation 10G versions. At the same time, SARDANA aims to reduce the complexity of the network infrastructure by implementing a fully passive (no power supply) plant from the $\mathrm{CO}$ to the end user premises, with several RNs interconnecting the WDM ring with the TDM trees.

This means a zero power consuming plant that focalize the hot spots only in the $\mathrm{CO}$ and at the end user premises where are present low cost devices. By simply adopting an intelligent OLT location with both a capillary fiber optic diffusion and the implementation of a regional/sub regional SARDANA infrastructure would be, in principle, possible to reduce the number of CO from 820-11, thus having a tremendous impact on the carbon footprint, as well as on the operator maintenance costs reduction.

Figure 2 and 3 show the scheme and the main results obtained during the First SARDANA integration experiment. The network has been tested in a $105 \mathrm{~km}$ long ring between Rome and Pompeii cities, at 10G DS (down stream) and 2.5G US (up stream), with 2 RNs and 3 optical channels. The pump power at the $\mathrm{CO}$ was below 1.2 watts at $1480 \mathrm{~nm}$. Sensitivities are -33 and $36 \mathrm{dBm}$ respectively. Error free conditions as well as protection against fiber cut was validated, with less than $1 \mathrm{~dB}$ penalty at rerouting, in DS and US directions.

\section{RESULTS}

Energy efficiency of the sardananetwork: In order to evaluate the energy efficiency of the SARDANA network, we developed a model for estimation of power consumption of various long-reach optical access networks (Lovric et al., 2011a; 2011b; Lovric and Aleksic, 2010a; 2010b). All considered networks are FTTH variants (Lam, 2007) including various realizations of passive optical networks (GPON ITUTT Recommendation 2008 10G-EPON IEEE 802.3av, Wavelength-Division Multiplexing PON (WDM PON), optical Point-To-Point (P-t-P) Ethernet based networks (1 Gbit $\sec ^{-1}-1 \mathrm{GE}$ and 10 Gbit $\left.\sec ^{-1}-10 \mathrm{GE}\right)$ and the hybrid ring-tree TDM/WDM PON as proposed in the SARDANA project. We considered reach extension of access networks by means of optical amplifiers Doped Fiber Amplifier (DFA) and Semiconductor Optical Amplifier (SOA) and optoelectronic repeaters (O/E/O).

The energy efficiency of the whole network was estimated by taking into account all contributing elements and peculiarities of each technology such as topology, typical configuration and realization and maximum Data rates in Down Stream (DS) and Up Stream (US) directions. Additionally, the achievable bandwidth per subscriber is calculated as a function of the number of subscribers, the maximum available DS and US data rates and the aggregated uplink throughput in the Central Office ( $\mathrm{CO}$ ) of a network provider. The 
network elements are modeled at the chip level. That means first generic structures for both network-side and user-side elements are specified and then their total power consumption is calculated by summing up values for consumption of individual functional blocks. The $\mathrm{CO}$ equipment is modeled as an aggregation switch with a number of downlink ports, Ethernet or PON ports and a number of $10 \mathrm{G}$ Ethernet uplink ports. Other important parameters are the number of users per $\mathrm{CO}$ and the uplink capacity of a $\mathrm{CO}$. The combination of the above mentioned parameters and their values represent the input to the model, whilst the output of the model is energy efficiency obtained from the power consumption values of the whole network and the resulting achievable data rates per user. For a more detailed description of the model and parameters used please refer to (Lovric et al., 2011; Aleksic and Lovric, 2010). Here, we define energy efficiency as the number of bits that can be transferred through the network per unit of energy consumed, which is then presented in $\mathrm{bit} / \mathrm{J} \equiv(\mathrm{bit} / \mathrm{s}) / \mathrm{W}$.

We assume three scenarios with different uplink bandwidths. The first scenario envisages an unlimited uplink in the CO. It can be seen as a theoretical scenariofor long-term future, where metropolitan area networks will be able to provide a large enough capacity so that there will be no practical bandwidth limitation for the considered access technologies. Thus, each access option can provide the maximum link data rate to each subscriber. The second scenario considers a limited uplink of $400 \mathrm{Gbit} \mathrm{sec}^{-1}$, while in the third scenario there is a more strong limitation of $100 \mathrm{Gbit}$ $\mathrm{sec}^{-1}$. Additionally, we look at three different sizes of the access network with $100,1,000$ and 10,000 subscribers connected to a single $\mathrm{CO}$. The considered data rates for different technologies are net line data rates excluding the line coding overhead. For instance in the case of P-t-P $1 \mathrm{GE}$ we assume $1 \mathrm{Gbit} \mathrm{sec}^{-1}$, which is the line data rate without the overhead associated with the $8 \mathrm{~B} / 10 \mathrm{~B}$ coding. In the case of PONs, we assume that every user can receive the maximum downstream data rate, while upstream data rate is shared among all users connected to a particular OLT, i.e., to a TDM tree. For P-t-P networks, we assume that all subscribers can achieve the maximum data rate as long as it is not limited by the maximum capacity of the aggregation switch to which they are connected and by the maximum uplink capacity of the corresponding CO.

For the SARDANA network, we assume 32 wavelength channels, each with a TDM tree comprising 32 ONUs, which could provide access to 1024 users. SARDANA provides $10 \mathrm{Gbit} \mathrm{sec}^{-1}$ of DS and $2.3 \mathrm{Gbit}$ $\mathrm{sec}^{-1}$ of US net data rate per TDM tree. In our power efficiency analysis, we introduce two SARDANA cases. The first one (SARDANA 1) refers to the existing test bed in which values of power consumption are real measured values of the devices that are deployed in the test bed. The power consumption of the test bed equipment is not optimized in terms of power savings and improved efficiency as it would be in some degree done in commercial equipment. In the second case (SARDANA 2), we assume that the network is equipped with market ready components, i.e., with components which canprovide required performance and are optimized for deployment in the field. Such components would provide remarkably lower power consumption. However, electronic devices for $10 \mathrm{G}$ PONs are still hardly commercially available, therefore we collected available data about power consumption and formed a database that refers to many scientific studys and some product data sheets. According to those values, we were able toestimate the power consumption of future 10G PON devices that could be used for SARDANA.

The estimated energy efficiency of the two SARDANA cases in comparison to five other FTT H networks is shown in Fig. 4-6. In the first scenario with unlimited capacity of the metropolitan area network (Fig. 4), the energy efficiency remains almost the same for all three sizes of the network. Independent on the number of subscribers, the high-speed options such as 10G-EPON, P-t-P 10G Ethernet and SARDANA 2 provide for a factor between 3 and 4 higher efficiency than P-t-P $1 \mathrm{G}$ Ethernet and $1 \mathrm{Gbit} \mathrm{sec}^{-1}$ WDM PON. GPON and the SARDANA test bed (SARDANA 1) provide moderate efficiencies of about 150 and 215 Mbit per Joule, respectively. For a small network with 100 subscribers only, the efficiency of TDM and hybrid TDM/WDM PON options is not affected by the uplink limitation, while the P-t-P and WDM PON options, especially the high-speed ones such as P-t-P $10 \mathrm{GE}$, become the least efficient ones because the potentially high data rate per user cannot be achieved anymore.

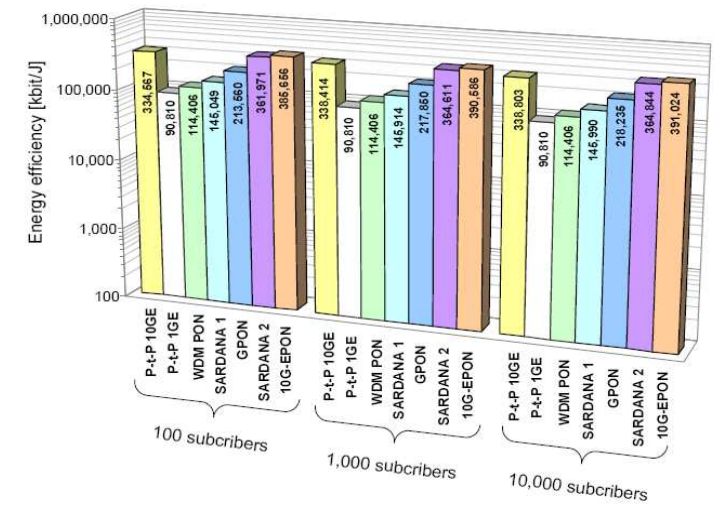

Fig. 4: Energy efficiency of the considered optical access networks with unlimited uplink 


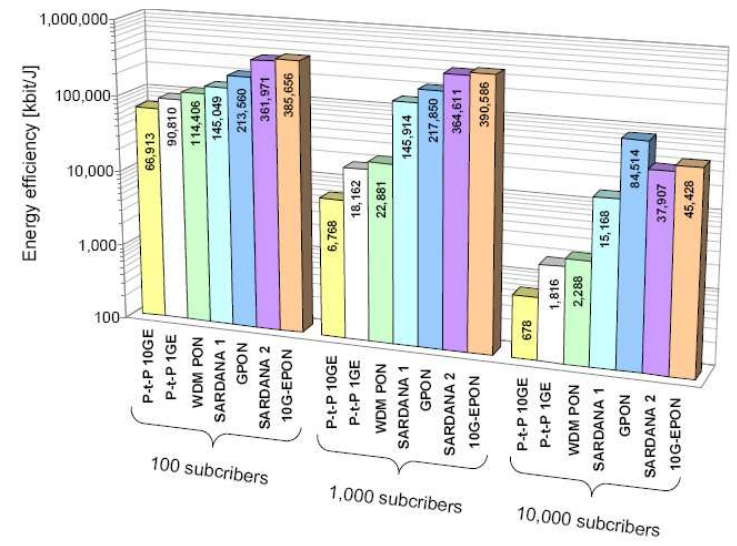

Fig. 5: Energy efficiency of the considered optical access networks with a limited uplink of $400 \mathrm{Gbit}$ $\sec ^{-1}$

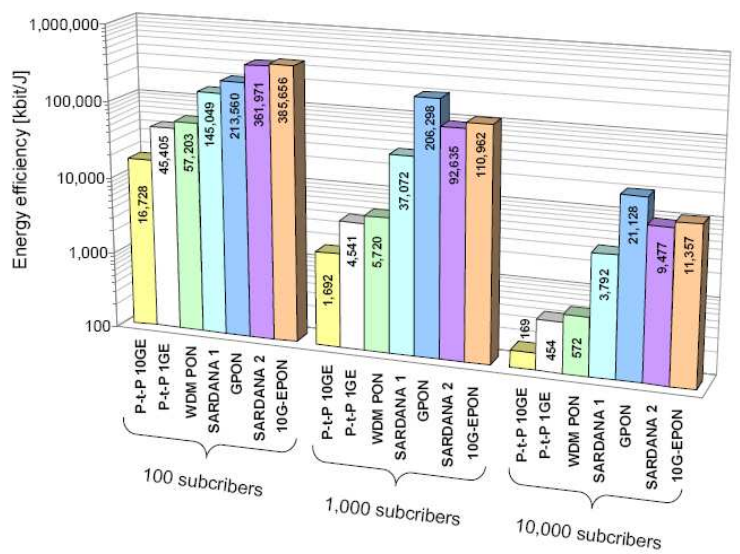

Fig. 6: Energy efficiency of the considered optical access networks with a limited uplink of 100 Gbit $\sec ^{-1}$

When increasing the number of users and reducing the uplink bandwidth, the difference in energy efficiency between TDM and PONs and P-t-P FTTH networks increases.

For a 100 Gbit $\sec ^{-1}$ uplink and 10,000 subscribers per CO (Fig. 6), one Joule of energy is consumed for transferring approx. 170 kbit of data through a P-t-P 10GE network, while 125 times more data can be transferred through a GPON-based network for the same energy.

\section{DISCUSSION}

Optical technologies can be a real solution to both lower the power consumption impact and to deliver the real broadband to the end user. The diffusion of Passive Plants (PON) and the intelligent distribution of the Central Offices (CO) on the territory become crucial to deliver flexible and scalable networks reducing at the same time the carbon footprint. Thus, the most efficient option for high number of subscribers and strongly limited uplink is GPON followed by 10GEPON and SARDANA 1. In general, SARDANA provides very good energy efficiency for both limited and unlimited uplink and for small and large etworks.

\section{CONCLUSION}

The SARDANA project demonstrated that is possible to reduce the $\mathrm{CO}$ number, to increase the end users served and to expand the reach beyond the GPON limits lowering the overall consumption by adopting an intelligent amplification strategy.

\section{ACKNOWLEDGMENT}

Study described in this study was carried out with the support of the SARDANA Project and the BONE project ("Building the Future Optical Network in Europe"), both funded by the European Commission through the 7th ICT Framework Programmed. The authors kindly acknowledge the COST IC0802 Action and the ATENA project.

\section{REFERENCES}

Aleksic, S., 2009. Energy-efficient global networks and their implications. Proceedings of the 10th International Conference on Telecommunications, Jun. 8-0, IEEE Xplore Press, Zagreb, pp: 331-338.

Aleksic, S. and A. Lovric, 2010. Power efficiency in wired access networks: Energieeffizienz in drahtgebundenen zugangsnetzen. I E Elec. Comput. Eng., 127: 321-326. DOI 10.1007/s00502-010-0783-5

Lam, C.F., 2007. Passive Optical Networks: Principles and Practice. 1st Edn. Academic Press, Amsterdam, Boston, ISBN-10: 0123738539, pp: 324.

Lovric, A. and S. Aleksic, 2010a. Influence of uplink limitation and broadcast traffic on power efficiency in long-reach optical networks. Proceedings of Asia Communications and Photonics Conference Exhibition, Dec. 8-12, IEEE Xplore Press, Shanghai, pp: 735-736. DOI: 10.1109/ACP.2010.5682713 
Am. J. Engg. \& Applied Sci., 4 (4): 485-490, 2011

Lovric, A. and S. Aleksic, 2010b. Power efficiency of extended reach 10G-EPON and TDM/WDM PON. Proceedings of the Optical Fiber Communication (OFC), Collocated National Fiber Optic Engineers Conference, Mar. 21-25, IEEE Xplore Press, San Diego, pp: 1-3.

Lovric, A., S. Aleksic, J.A. Lazaro, G.M.T. Beleffi and F. Bonada et al., 2011a. Influence of broadcast traffic on energy efficiency of long-reach SARDANA access network. Proceedings of the National Fiber Optic Engineers Conference Optical Fiber Communication Conference and Exposition, Mar. 6-10, IEEE Xplore Press, Los Angeles, California, pp: 1-3.
Lovric, A., S. Aleksic, J.A. Lazaro, G.M. Tosi Beleffi and J. Prat et al., 2011b. Power efficiency of SARDANA and other long-reach optical access networks. Proceedings of the 15th International Conference on Optical Network Design and Modeling, Feb. 8-10, IEEE Xplore Press, Bologna, pp: 1-6. 